\title{
CIÊNCIA, TECNOLOGIA E SOCIEDADE: PERSPECTIVAS MULTIDISCIPLINARES SOB ENFOQUES TEÓRICOS E APLICADOS
}

LEITE, Ana Cláudia de Oliveira. Bibliotecária com especialização em Gestão Organizacional e Recursos Humanos e MBA em Gestão de Unidades de Informação. Mestranda no Programa de Pós-Graduação em Ciência, Tecnologia e Sociedade e tutora virtual nos cursos de graduação e pós-graduação na UAB/ Universidade Federal de São Carlos - UFSCar. Rua Ignácio Florêncio Silveira, 3258, Vila Paulista, Pirassununga (SP). CEP: 13.632-210. E-mail: anadeoliver@ yahoo.com.br.

\begin{abstract}
Resumo
Considerando-se a emergência do campo de Ciência, Tecnologia e Sociedade, o trabalho apresenta uma resenha sobre duas obras publicadas pela Pedro e João Editores: Ciência, Tecnologia e Sociedade: perspectivas multidisciplinares, organizado por Valdemir Miotello e Wanda Aparecida Machado Hoffmann, e Ciência, Tecnologia e Sociedade: enfoques teóricos e aplicados, organizado por Cidoval Morais de Souza e Maria Cristina Piumbato Innocentini Hayashi, que, ao discutirem pesquisas teóricas e práticas, assim como, estudos de caso de diversas áreas do conhecimento, contribuem para a construção de um diálogo aberto e multidisciplinar sobre as dimensões sociais do desenvolvimento científico e tecnológico e suas influências no mundo contemporâneo.
\end{abstract}

Palavras-chave: Ciência; Tecnologia e Sociedade; Pesquisa científica; Pós-graduação.

\begin{abstract}
Considering the emergence of the field of Science, Technology and Society, this paper presents a review of two books published by Pedro e João Editores: Ciência, Tecnologia e Sociedade: perspectivas multidisciplinares, organized by Valdemir Miotello and Wanda Aparecida Machado Hoffmann, and Ciência, Tecnologia e Sociedade: enfoques teóricos e aplicados, organized by Cidoval Morais de Souza and Maria Cristina Piumbato Innocentini Hayashi, that discuss theoretical and practical researches and case studies from different fields of knowledge, and contribute to build an open and multidisciplinary speech about the social dimensions of scientific and technological development and its influences on contemporary world.
\end{abstract}

KEYWORDs: Science, technology and society; Scientific research; Graduate work. 
As transformações ocorridas no século $\mathrm{XX}$, advindas do desenvolvimento científico, tecnológico, social e econômico, suscitaram reflexões e críticas acerca do real papel da ciência e da tecnologia (C\&T) na sociedade. A partir das décadas de 1960 e 1970, tais discussões eclodiram no surgimento do Movimento Ciência, Tecnologia e Sociedade (CTS), o qual buscava a desmistificação da $C \& T$ e a crítica à sua neutralidade.

No Brasil, alguns institutos de pesquisa e instituições de ensino superior investigam os aspectos sociais da C\&T como, por exemplo, o Programa de PósGraduação em Ciência, Tecnologia e Sociedade (PPGCTS) da Universidade Federal de São Carlos (UFSCar). Esse programa é composto por três linhas de pesquisa ("Dimensões Sociais da Ciência e da Tecnologia", "Gestão Tecnológica e Sociedade Sustentável" e "Linguagens, Comunicação e Ciência"), que possibilitam a interação entre docentes e discentes de diferentes áreas do conhecimento.

Em 2008, dentre os projetos desse programa, destacou-se a publicação de dois livros pela Pedro e João Editores: Ciência, Tecnologia e Sociedade: perspectivas multidisciplinares, organizado por Valdemir Miotello e Wanda Aparecida Machado Hoffmann, e Ciência, Tecnologia e Sociedade: enfoques teóricos e aplicados, organizado por Cidoval Morais de Souza e Maria Cristina Piumbato Innocentini Hayashi. Essas obras constituem uma interessante colaboração na investigação do fenômeno científico-tecnológico no contexto social.

A primeira obra, escrita por professores do PPGCTS e convidados, revela a complexidade do campo CTS em nove capítulos: no primeiro capítulo, Renato Dagnino discute a análise da política no campo CTS e seus modelos cognitivos. No segundo capítulo, Maria Cristina Piumbato Innocentini Hayashi e Ariadne Chloë Mary Furnival apresentam um panorama do campo CTS no Brasile suas principais tendências. No terceiro capítulo, Wanda Aparecida Machado Hoffmann, Leandro Innocentini Lopes de Faria e José Ângelo Rodrigues Gregolin abordama importância dos estudos de prospecção tecnológica para a compreensão e planejamento de programas em C\&T.
No quarto capítulo, Rômulo Marinho do Rêgo, Rogério Gaudêncio do Rego e Cidoval Morais de Souza relacionam o ensino de ciências na educação básica e a cultura popular sob a abordagem CTS. No quinto capítulo, Roberto Carlos dos Santos Pacheco, Vinícius Medina Kern e Andréia Valéria Steil analisam a contribuição das plataformas Egov de informação em ciência, tecnologia e inovação para a tomada de decisão para o desenvolvimento regional. No sexto capítulo, Maria Teresa Miceli Kerbauy aborda a descentralização e a inovação das políticas sociais no Brasil e seus impactos no desenvolvimento regional. No sétimo capítulo, Isabel Cristina dos Santos e João Amato Netto apresentam um estudo de caso sobre a formação do polo aeronáutico na cidade de São José dos Campos (SP) e seus reflexos na comunidade local. No oitavo capítulo, Edilson Pepino Fragalle investiga os Centros de Pesquisa, Inovação e Difusão, localizados no polo tecnológico de São Carlos (SP) e o processo de gestão da informação científica e da comunicação para o desenvolvimento regional. E, por fim, no último capítulo, Márcia Niituma Ogata e Wilson José Alves Pedro discutem um estudo de caso sobre a gestão de recursos humanos em saúde e as concepções de ciência, tecnologia e inovação.

A segunda obra, escrita por alunos do PPGCTS, reúne uma diversidade de ideias e argumentos em 14 capítulos: no capítulo 1, Ana Paula Meneses Alves propõe uma reflexão sobre a comunicação científica e os periódicos científicos pelo olhar CTS. No capitulo 2, Aparecida Zem Lopes discute a importância do campo CTS na compreensão e fortalecimento de arranjos produtivos locais, com o estudo de caso da indústria calçadista em Jaú (SP). No capítulo 3, Caroline Periotto, aborda a questão da gestão da informação e a sua utilização em empresas de base tecnológica. No capítulo 4, Ednéia Silva Santos-Rocha apresenta indicadores bibliométricos da Revista Iberoamericana de Ciencia, Tecnologia y Sociedad. No capítulo 5, Fabricio Mazocco relata algumas tendências da comunicação pública da ciência. No capítulo 6, Fernanda de Freitas reflete sobre a linguagem audiovisual como mediadora de discursos 
científicos sobre a loucura. No capítulo 7, Guilherme Guimarães Pallerosi analisa a construção social das tecnologias de baixo impacto ambiental com um estudo de caso do polo petroquímico da região do $\mathrm{ABC}$ paulista. No capítulo 8, Helen Cristina Pedrino relaciona ciência, tecnologia e inovação em saúde, sob o enfoque CTS. No capítulo 9, Igor José Siquieri Savenhago demonstra que cartas de presidiárias e expresidiárias podem ser analisadas como documentos sob o olhar da ciência, com o relato de um projeto desenvolvido na Penitenciária Feminina de Ribeirão Preto (SP). No capítulo 10, Jandira Rossi faz uma revisão dos softwares de apoio à gestão da informação em atividades de inteligência competitiva e prospecção tecnológica. No capítulo 11, Márcia Ferreira Pinto discute as mudanças de paradigma do setor sucroalcooleiro. No capítulo 12, Mateus Yuri Ribeiro da Silva Passos aponta a inter-relação entre jornalismo literário e a comunicação pública da ciência, sob as perspectivas do campo CTS. No capítulo 13, Patrícia Villar Martins apresenta um estudo de caso daAgência de Inovação da UFSCar. E no capítulo 14, Priscilla Oliveira promove uma discussão sobre a cultura organizacional e a inovação.

Considerando-se a emergência do campo CTS, as duas obras constituem ricas fontes de informação, que apresentam pesquisas teóricas e práticas, assim como estudos de caso de diversas áreas do conhecimento, na construção de um diálogo aberto e multidisciplinar. Portanto, a sua contribuição é fundamental na consolidação do campo CTS no país e sua leitura é recomendada àqueles que queiram compreender as dimensões sociais da C\&T e suas influências no mundo contemporâneo.

\section{REFERÊNCIAS}

MIOTELLO, V.; HOFFMANN, W. A. M.

Perspectivas Multidisciplinares em Ciência, Tecnologia e Sociedade. São Carlos: Pedro e João Editores, 2008. 297 p.

SOUZA, C. M. (Org.); HAYASHI, M. C. P. I. (Org.) Ciência, Tecnologia e Sociedade: enfoques teóricos e aplicados. São Carlos: Pedro e João Editores, 2008. 296 p.

RECEBIDO EM 16/11/2010

ACEITo EM 10/12/2010 